\title{
CUALIDADES Y COMPETENCIAS DEL PROFESOR UNIVERSITARIO: LA VISIÓN DE LOS DOCENTES
}

\author{
QUALITIES AND COMPETENCES OF \\ THE UNIVERSITY PROFESSOR: \\ THE TEACHERS' VISION SUMMARY
}

\author{
Sara Elvira Galbán Lozano ${ }^{1}$ \\ https://orcid.org/0000-0002-9915-0441
}

Claudia Fabiola Ortega Barba ${ }^{2}$

https:// orcid.org/0000-0003-2709-2024

Recibido: septiembre, 302020 - Aceptado: octubre 19, 2020

\section{RESUMEN}

Este artículo es producto de una investigación cuyo objetivo fue caracterizar al buen profesor universitario a partir de las consideraciones que este hace en torno a su propia práctica. Para cumplir el objetivo, se optó por el enfoque cualitativo con perspectiva fenomenológica, utilizando a la entrevista semiestructurada como técnica de recopilación de información, y al método de las comparaciones constantes para el proceso de análisis. Dentro de los hallazgos más relevantes se encontró que los profesores participantes, todos ellos experimentados, consideran que un buen docente universitario necesita de cualidades personales, de competencias técnicas, de pasión por enseñar, del buen empleo de estrategias didácticas centradas en el aprendizaje pero, que lo más importante, radica en colocar en el centro de la actividad profesional al estudiante.

Palabras clave: competencias del docente, enseñanza, interés por el aprendizaje, investigación pedagógica, profesor de enseñanza superior.

1 Doctora en Pedagogía, Universidad de Barcelona, España. Profesora investigadora, Escuela de Pedagogía, Universidad Panamericana, México. Candidata en el Sistema Nacional de Investigadores.sgalban@up.edu.mx

2 Profesora investigadora, Escuela de Pedagogía, Universidad Panamericana, México. Doctora en Pedagogía, Universidad Nacional Autónoma de México. Candidata en el Sistema Nacional de Investigadores. cortega@up.edu.mx 


\begin{abstract}
This research shows the characteristics of a good university professor from the perspective they have about their own practice. To meet the objective, we opted for the qualitative approach with a phenomenological perspective, using the semi-structured interview as an information gathering technique and the method of constant comparisons for the analysis process. Among the most relevant findings is that all of the professors consider that a good university teacher needs both personal qualities and technical competences, but that the most important consideration should be a student centered education process, along with passion for teaching and the use of didactic strategies focused on learning.
\end{abstract}

Keywords: Teacher Competences, Teaching, Interest in Learning, Pedagogical Research, Higher Education Teacher.

\title{
INTRODUCCIÓN
}

En las publicaciones especializadas sobre docencia universitaria es una constante el preguntarse sobre qué diferencia a un profesor, bien valorado por los estudiantes, de otro que no cumple con las expectativas de los mismos; una de las respuestas se encuentra en las competencias que este profesional ha de tener, como lo afirma Mas (2012): la calidad del profesor en las aulas no se reduce al dominio de los contenidos de su área de especialización, sino que requiere de saberes pedagógicos, didácticos, curriculares, contextuales y prácticos (Jarauta y Medina, 2012). Ello se refuerza con el trabajo de Alonso (2019) quien comenta que un buen profesor universitario es aquel con cualidades personales, profesionales y metodológicas, siendo las más valoradas por los estudiantes: la claridad en la exposición, el dominio de la asignatura, la buena comunicación y preparación de las clases, junto con el respeto, la actitud positiva y la calidad humana.

Ser un buen profesor universitario significa mucho más que el solo poseer la capacidad intelectual para utilizar estructuras cognitivas, como base para desarrollar el proceso enseñanza-aprendizaje; el serlo implica un camino integral de mejora personal con el fin de promover la transformación del que aprende, lo cual no se resuelve solo con el dominio del contenido y de los medios didácticos, sino sobre todo mediante la preocupación de cuidar al otro, de procurarle el mayor bien posible, de cubrir sus necesidades. Esto requiere el cultivo de cualidades personales y profesionales con miras a influir en el estudiante y motivarlo hacia la acción educativa (Galbán, 2016; Meza, 2017).

El objetivo de la presente investigación es mostrar las características del buen profesor universitario a partir de las consideraciones que este hace en torno a su propia práctica. Lo anterior resulta significativo para la investigación sobre docencia universitaria pues, en general, los estudios que rescatan la idea de caracterizar a un buen profesor lo hacen, por un lado, a partir de las valoraciones que los estudiantes realizan sobre la práctica docente, y por el otro, en las percepciones que tienen los profesores durante su formación inicial, dejando muchas veces de lado a los profesores experimentados.

\section{EL PROFESOR UNIVERSITARIO}

La universidad se concibe como «una comunidad de personas y saberes, como una institución dedicada a la enseñanza, al cultivo del saber universal, a su acrecentamiento 
mediante la investigación en las diversas ciencias, y a la educación superior e integral de sus estudiantes» (Ponz, 1996: 86), convirtiéndose en la expresión corporativa de la vocación de enseñar y del deseo de aprender.

La tarea fundamental de cultivar el saber, define a la universidad como organización y espacio de relaciones sociales, de diálogo. Su eje de sustentación es la cultura que requiere como condición necesaria del pensamiento y de la búsqueda de la verdad, mediante la investigación y la docencia (Hernández, 2018); ahí surge el profesor universitario, concebido como un estudioso, permanente generador de conocimiento y de comunidad académica, un visionario y conductor de un complejo proceso de formación, quien requiere poseer ciencia, experiencia y vocación académica, además de integridad moral y espíritu indagador (Hernández, 2018). Todo ello configura el rol docente que, según Zabalza (2002), se expresa en dos dimensiones: personal y profesional.

En la primera, parece claro que buena parte de la capacidad de influencia de los profesores en los estudiantes, se deriva de lo que se es como persona; por eso, si el profesor está satisfecho con su trabajo y posee una trayectoria académica bien trazada, mejoran los procesos formativos, lo que además lleva a reconocer la docencia como profesión enmarcada en un área del saber especializado (dimensión profesional), en donde «no es suficiente dominar los contenidos, ni ser un buen investigador en el campo, la profesionalidad docente tiene que ver con los alumnos y cómo podemos actuar para que aprendan efectivamente lo que pretendemos enseñarles» (Zabalza, 2002: 114). De este rol se desprenden distintas funciones institucionales: investigación, docencia, difusión de las investigaciones y de las innovaciones pedagógicas, tutoría y participación en la gestión académica (Benedito, 1992; Mas, 2012).

También de funciones propias de un microescenario -el aula- que se concretan en la formación del estudiante en relación con el objeto disciplinar y, por el otro, en la preparación del alumno para desenvolverse en el terreno profesional y humano (Pérez, Ruiz y Sanz, 2014). Esto exige del profesor universitario, ayudar y animar a los estudiantes a aprender para lo cual se requiere, según Bain (2007) y Tarabay (2009), de las siguientes cualidades: dominar el contenido que se busca enseñar; basar las sesiones en la resolución de problemas y en retos intelectuales; conformar entornos para el aprendizaje crítico natural; confiar y acoger a los estudiantes; evaluar continuamente la práctica docente, y tener vocación por la enseñanza.

Paralelamente a estas cualidades, las competencias docentes «se caracterizan por ser complejas, pues combinan habilidades, principios y conciencia del sentido y de las consecuencias de las prácticas pedagógicas; así como una reflexión y análisis sobre los contextos que las condicionan y que van más allá del aula» (Barrón, 2009: 78); se refieren no solo al capital de conocimientos disponibles, sino a habilidades profesionales y valores éticos, todo ello vinculado con el saber, el saber hacer, el saber ético y el saber convivir (Durán, 2016).

En la literatura especializada, existen diversas clasificaciones sobre competencias docentes, que buscan configurar el perfil del profesor universitario, con miras a establecer procesos de formación y evaluación. Referente obligado es la propuesta de Zabalza (2007), quien señala que un buen profesor universitario debe: planificar el proceso de enseñanza aprendizaje; seleccionar y preparar los contenidos disciplinares; ofrecer explicaciones comprensibles; manejar tecnología; diseñar metodología y organizar las actividades; comunicarse-relacionarse con los alumnos; asesorar y evaluar; reflexionar e investigar sobre la enseñanza; identificarse con la institución, y trabajar en equipo. 
Otra propuesta sobre competencias del docente universitario es la desarrollada por Mas (2011) quien establece cinco: 1) diseñar el programa de la asignatura de acuerdo a necesidades, contexto y perfil profesional; 2) desarrollar el proceso didáctico, promoviendo oportunidades de aprendizaje individual y grupal; 3) asesorar el proceso de aprendizaje de los alumnos, propiciando la autonomía; 4) evaluar el proceso de enseñanza aprendizaje, y 5) contribuir activamente a la mejora de la docencia.

Por su parte Ruiz y Aguilar (2017), a partir de la elaboración y validación de un cuestionario de autoevaluación de la práctica docente, establecen dos tipos de competencias del docente universitario: transversales y específicas. Las primeras atraviesan todo el trabajo docente y se refieren a valores, actitudes y cualidades personales que impregnan la actividad cotidiana, además de reflejar la identidad y los principios de la institución educativa en la que se trabaja. Las segundas, se relacionan con las funciones propias del profesor universitario: docencia, investigación, vinculación con la sociedad y gestión institucional.

Pareciera, pues, que ser un buen profesor universitario implica cumplir ciertas funciones y poseer conocimientos, cualidades y competencias a las cuales se ha hecho mención; sin embargo, al ser la profesión docente compleja y dinámica es necesario escuchar la voz de los actores a partir de su experiencia y lo que estos consideran los hace ser reconocidos por los estudiantes.

\section{METODOLOGÍA}

Esta investigación se enmarca en el paradigma interpretativo y el enfoque cualitativo, con perspectiva fenomenológica. Como afirman Merriam y Tisdell (2016), los estudios cualitativos «[...] buscan descubrir y comprender un fenómeno, un proceso o las perspectivas y cosmovisiones de las personas involucradas, cómo las personas interpretan sus experiencias y qué significado les atribuyen» (p. 15). En este caso, se busca mostrar las características del buen profesor universitario a partir de sus consideraciones sobre la práctica docente; lo que interesa es resaltar la mirada sobre su propia experiencia y lo que él considera lo hace ser bien valorado por sus estudiantes.

Se optó por una perspectiva fenomenológica: conocer la realidad tal como otros la experimentan, para entender cómo perciben las cosas los protagonistas (Taylor y Bogdan, 1994). Patton (2015) explica que la fenomenología busca comprender cómo las personas describen e interpretan las experiencias, de esta forma se examina el modo en que otros experimentan el mundo. Importa lo que las personas perciben como relevante, pues se considera preferente el significado que los propios protagonistas otorgan a sus acciones (Tójar, 2006). Así mismo, desde esta perspectiva, los seres humanos están vinculados con su mundo y, por ende, enfatiza la experiencia vivida, la cual aparece en el contexto de las relaciones con objetos, personas, sucesos y situaciones (Galeano, 2004).

\section{CONTEXTO Y PARTICIPANTES}

El criterio de inclusión en este estudio remite a profesores universitarios adscritos a diferentes escuelas y facultades de una universidad particular de la Ciudad de México, reconocidos por los estudiantes como profesores de excelencia en la evaluación semestral 
institucional que valora el desempeño docente. Dicha evaluación mide, a través de una escala estimativa, rubros en torno a la identificación con la filosofía institucional, así como a las cualidades y competencias docentes de los profesores.

A continuación se muestra el perfil de los once profesores participantes en este estudio.

Figura 1. Perfil de los profesores

\begin{tabular}{|c|c|c|c|c|c|c|c|}
\hline Profesor & Sexo & Edad & Escolaridad & $\begin{array}{c}\text { Área de } \\
\text { estudio }\end{array}$ & $\begin{array}{c}\text { Área de } \\
\text { adscripción }\end{array}$ & $\begin{array}{c}\text { Años de } \\
\text { docencia }\end{array}$ & $\begin{array}{c}\text { Tipo de } \\
\text { contratación }\end{array}$ \\
\hline P1 & Masculino & 31 & Licenciatura & Pedagogía & Pedagogía & 6 & Asignatura \\
\hline P2 & Femenino & 54 & Doctorado & $\begin{array}{c}\text { Terapia en } \\
\text { comunicación } \\
\text { humana }\end{array}$ & Pedagogía & 30 & Tiempo completo \\
\hline P3 & Femenino & 53 & Maestría & Pedagogía & Empresariales & 28 & Asignatura \\
\hline P4 & Masculino & 45 & Maestría & Derecho & Derecho & 20 & Asignatura \\
\hline P5 & Masculino & 40 & Doctorado & $\begin{array}{c}\text { Administración } \\
\text { de empresas }\end{array}$ & Empresariales & 15 & Tiempo completo \\
\hline P6 & Masculino & 45 & Doctorado & $\begin{array}{c}\text { Derecho } \\
\text { Comunicación }\end{array}$ & 22 & Tiempo completo \\
\hline P7 & Femenino & 50 & Maestra & $\begin{array}{c}\text { Administración } \\
\text { de empresas }\end{array}$ & Empresariales & 25 & Asignatura \\
\hline P8 & Masculino & 78 & Doctorado & Filosofía & Filosofía & 50 & Asignatura \\
\hline P9 & Masculino & 58 & Licenciado & $\begin{array}{c}\text { Mecánico } \\
\text { electricista }\end{array}$ & Ingeniería & 30 & Tiempo completo \\
\hline P10 & Femenino & 38 & Maestría & $\begin{array}{c}\text { Terapia en } \\
\text { comunicación } \\
\text { humana }\end{array}$ & Psicología & 14 & Tiempo completo \\
\hline P11 & Femenino & 50 & Maestría & $\begin{array}{c}\text { Electrónica } \\
\text { Ingeniería }\end{array}$ & 25 & Asignatura \\
\hline
\end{tabular}

Fuente: Elaboración propia.

En el estudio participaron 5 mujeres y 6 hombres, con una media de edad de 45 años y de 22 años de tiempo en la docencia. Uno solo tiene licenciatura, seis cuentan con maestría y cuatro con doctorado, de estos últimos, dos pertenecen al Sistema Nacional de Investigadores. En cuanto al tipo de contratación, cinco trabajan de tiempo completo en la universidad y seis son de asignatura con tiempo de dedicación parcial a la docencia, pues atienden otros proyectos de sus campos de especialidad. 


\section{RECOPILACIÓN DE LA INFORMACIÓN}

Se empleó la técnica de la entrevista semiestructurada, basada en las siguientes ideas detonadoras: ¿por qué decidiste ser profesor universitario?, ¿cómo te concibes como profesor?, descripción de la práctica docente, fortalezas, áreas de oportunidad y retos como profesor.

Se realizaron once entrevistas de forma individual, pues como sugiere Robles (2011), esto establece un lazo de intimidad y complicidad con cada entrevistado de forma que comparten su experiencia con mayor confianza. Las entrevistas duraron entre 40 y 50 minutos y fueron grabadas en audio para su posterior transcripción, atendiendo al criterio de confidencialidad, con el permiso de los entrevistados.

\section{Análisis de la información}

Aun cuando el método cualitativo elegido es la fenomenología, se analizó la información con la propuesta surgida desde la teoría fundamentada (Strauss y Corbin, 1998) que sugiere el método de las comparaciones constantes. La anterior decisión se tomó debido a que:

[...] la naturaleza de las cuestiones de investigación, guía y orienta el proceso de indagación y, por tanto, la elección [y combinación] de unos métodos u otros [...], los métodos de investigación surgen bajo las concepciones y necesidades de los investigadores que trabajan desde una disciplina concreta del saber (Rodríguez, Gil y García, 1999: 40-41).

Este método combina la codificación inductiva de categorías con la comparación constante entre ellas, en tres momentos: la codificación abierta, la codificación axial y la codificación selectiva (Strauss y Corbin, 1998). Así, después de leer varias veces cada entrevista, se identificaron unidades de significado, las cuales fueron agrupadas en categorías de análisis, que se interrelacionaron, integraron y refinaron en un mayor nivel de abstracción (Creswell, 2002). Para llevar a cabo lo anterior se siguió un proceso inductivo y dialéctico que se describe a continuación.

En un primer momento, se realizó una lectura detallada de las entrevistas con el fin de tener una idea general de su contenido e identificar las principales temáticas en el discurso de los protagonistas. Posteriormente, se segmentó en unidades de significado, considerando los fragmentos que reflejaban ideas similares (codificación abierta). Cada unidad se estableció con un número consecutivo y un identificador del profesor participante (así P1 representa al primer profesor, P2 al segundo, hasta P11 al último).

A medida que avanzaba el análisis e iban apareciendo nuevas unidades de significado, se comparaban con las categorías previamente creadas para incluirlas en una de ellas; si alguna de las unidades no coincidía con las ya creadas, se definía una nueva categoría y se reubicaba (codificación axial). Finalmente, se depuraron los códigos creados y asignados a las unidades de significado, permitiendo fusionar categorías semejantes y redefinir algunas para adecuarse mejor al contenido.

Una vez que se hizo este procedimiento con cada entrevista y se alcanzó la saturación teórica (Strauss y Corbin, 1998) -es decir, que no aparecieron unidades de significado distintas a las ubicadas en las subcategorías, categorías y metacategorías ya configuradas, además de que estas contaban con información variada y suficiente-, se tomó la decisión de no realizar más entrevistas. 


\section{VALIDEZ Y CREDIBILIDAD}

Lincoln, Lynham y Guba (2011) proponen, como formas de rigor metodológico, el cuidado en la conceptualización, manejo de los datos y la presentación de resultados. Para establecer la validez y confiabilidad de los resultados se realizaron los procedimientos que a continuación se describen:

1) Una vez transcritas las entrevistas, los participantes tuvieron la oportunidad de revisarlas con la finalidad de aprobar o modificar la información. Esta técnica es descrita por Lincoln y Guba (1985) como la más importante para establecer credibilidad.

2) El proceso de categorización previamente descrito, fue realizado de manera colegiada por las autoras de esta investigación. Lo anterior hace referencia a lo que Patton (2015) sugiere como técnica de comparación que involucra a más de dos personas analizando datos y resultados.

\section{RESULTADOS}

En el análisis de la información emergieron dos metacategorías: 1) sobre la figura del profesor, y 2) sobre la práctica docente, dentro de las cuales se generaron categorías y subcategorías que se exponen a continuación:

Figura 2. Metacategorías, categorías y subcategorías del análisis

\begin{tabular}{|c|c|c|}
\hline Metacategorías & Categorías & Subcategorías \\
\hline \multirow{11}{*}{$\begin{array}{l}\text { Sobre la figura del } \\
\text { profesor. }\end{array}$} & \multirow{3}{*}{ Visión de sí mismo. } & Significado y papel docente. \\
\hline & & Dinamismo de la profesión docente. \\
\hline & & Relación con los estudiantes. \\
\hline & \multirow{6}{*}{ Cualidades. } & Pasión por enseñar. \\
\hline & & Confianza en el estudiante. \\
\hline & & Preocupación por el estudiante. \\
\hline & & Dominio del contenido. \\
\hline & & Exigencia académica. \\
\hline & & Gusto por el estudio. \\
\hline & \multirow[b]{2}{*}{ Competencias. } & Practicante reflexivo. \\
\hline & & Generador de ambientes de aprendizaje. \\
\hline \multirow{11}{*}{$\begin{array}{c}\text { Sobre la práctica } \\
\text { docente. }\end{array}$} & \multirow{3}{*}{ Planeación. } & Valoración. \\
\hline & & Orientaciones rectoras. \\
\hline & & Proceso. \\
\hline & Contextualización didáctica. & \\
\hline & Manejo de contenidos. & \\
\hline & \multirow{3}{*}{ Estrategias de enseñanza. } & Lecturas como fuente de reflexión. \\
\hline & & Intercambio de ideas. \\
\hline & & Reflexión sobre el aprendizaje. \\
\hline & $\begin{array}{l}\text { Variedad de medios } \\
\text { didácticos. }\end{array}$ & \\
\hline & \multirow[t]{2}{*}{ Evaluación. } & Retroalimentación. \\
\hline & & Corresponsabilidad profesor-alumno. \\
\hline
\end{tabular}

Fuente: Elaboración propia. 
Las características que muestran los profesores sobre su práctica docente se presentan de acuerdo con las metacategorías que emergieron durante el análisis de la información.

\section{Sobre la figura del profesor}

Esta metacategoría muestra información en torno a la percepción de los participantes sobre su ser profesional y se compone de tres categorías: visión de sí mismo, cualidades y competencias. En cuanto a la primera, los profesores expresan cómo se perciben frente a sus estudiantes, mientras que al hablar de cualidades y competencias, comparten diferentes aspectos en torno a lo que ellos consideran que los hace ser buenos profesores.

En referencia a la visión de sí mismo, las unidades de significado se agrupan en tres subcategorías; en un primer momento, uno de los profesores comparte la concepción sobre lo que le significa ser docente y su papel frente a los estudiantes, enfatizando su rol de aprendiente:

Yo creo, que es tener muy claro mi papel como docente. No transmito conocimientos: comparto conocimientos, ayudo a generar conocimientos y administro el conocimiento. Como docente soy un sujeto que aprende (P7).

Aunado a lo anterior, otro profesor menciona el dinamismo de la profesión al considerar que, a lo largo de su trayectoria, ha ido conformando su propio estilo docente:

Fui desarrollando mi propio método. Ahora ya lo tengo más claro: al principio me conflictuaba mucho, porque pensaba que tenía que hacer lo que hacía todo el mundo, que eso es lo que esperaban los alumnos de mí (P5).

En una tercera subcategoría agrupamos lo que los profesores expresan en cuanto a su forma de ser en relación con los estudiantes, destacando la cercanía, empatía y paciencia:

Intento ser cercano, intento ser exigente y estar continuamente reflexionando sobre mi práctica docente (P5).

Soy una persona empática; trato de conectar con mis alumnos. Soy una persona comprensiva, más no complaciente (P7).

No me cuesta trabajo que los estudiantes me entiendan y tengo mucha paciencia para resolver sus dudas las veces que sea necesario (P11).

Pasando a la categoría de las cualidades, los profesores expresan que uno de los primeros aspectos que los caracterizan es su pasión por enseñar, la cual consideran una fortaleza dentro de su práctica docente:

Me gusta mucho lo que hago, por eso estudio y leo mucho (P10).

Considero, como una de mis fortalezas, el amor a la docencia (P4).

Creo que soy un profesor que hace con pasión y con gusto lo que le toca hacer (P1).

La pasión por enseñar me hace mejor profesora, porque no es un «tengo que ir a clase»; a veces

llego cansada, pero trato de desconectarme y es como si el mundo se parara (P3).

Además de este amor por la enseñanza, los participantes manifiestan un interés por los estudiantes que se muestra en la confianza que les tienen, lo cual promueve el compromiso discente: 
Es increíble cómo se interesan en la asignatura, cómo prestan atención. Durante la clase no vuela ni una mosca. No tengo duda que es por la confianza que les tengo (P5).

Sí, he visto que trabajan motivados. [Por ejemplo] Pides como evidencias fotos... y te llegan con videos; entonces ves que dan más de lo que se les pide, porque confías en ellos. Eso me enriquece mucho como maestro (P1).

Lo anterior se ve reforzado por su preocupación por los estudiantes, vistos como personas y como futuros profesionistas, agentes de cambio, incluso de la práctica docente:

Una de mis fortalezas es el interés por cada uno de mis alumnos como seres humanos, sobre todo porque sean agentes de cambio (P3).

Hacer reflexionar a los estudiantes es una de mis mejores prácticas en el aula, porque la vida es más que una materia. Yo les digo: «Tu carrera es muy importante, pero tu vida no la define tu carrera sino tus valores; sin embargo, a través de esta te tienes que desarrollar y preparar, porque debes ser agente de cambio» (P11).

Yo creo que cualquier profesor tendría que ver, en su alumno, alguien que puede sacar lo mejor de uno (P6).

Esta preocupación se refleja en la atención personalizada que algunos manifiestan como una actividad cotidiana:

Me gusta mucho atenderlos personalmente, yo prefiero decirles que me busquen en mi oficina para explicarles los contenidos que se les dificultan (P11).

Si veo que a la tercera no entienden, les pido que me busquen después de la clase, porque a veces los alumnos están cansados y no pueden asimilar lo mismo, aunque les trates de explicar de tres maneras diferentes (P2).

Si ves que un alumno va mal, que va faltando, que no entrega tareas y lo dejas que siga así, me parece muy sádico, porque se va a hundir, si se va chueco, hay que ayudarlo a corregir (P8).

Siendo el estudiante la fuente principal de inspiración, los profesores resaltan la importancia de la exigencia académica, relacionándola con el dominio del contenido que imparten:

Mantengo un nivel alto, para lo cual conozco y domino muy bien las materias que doy (P9). Me describo como una profesora exigente que tiene muy claros los objetivos. Conozco, en general, muy bien la materia que imparto y cada uno de los temas (P3).

Dicho dominio es consecuencia de su gusto por el estudio y la actualización continua, como fuente de mejora personal y profesional:

Siempre me ha gustado mucho estudiar. Desde niño me gustaba leer y estudiar. Ahora ya no lo hago, sino que re-leo y re-estudio (P8).

Constantemente estoy inquieto por estudiar sobre buenas prácticas en la docencia, independientemente de los estudios formales que ya he realizado (P5).

Siento que tengo mucho que aprender todavía como docente. Evidentemente en contenidos siempre hay algo nuevo y seguramente también en materia pedagógica; ahí estoy siempre viendo en dónde flaqueo y lo estudio. Me gusta mucho estudiar (P4).

Relacionan también la exigencia académica con el establecimiento y cumplimiento de la normatividad de la clase: 
Yo tengo un nivel de exigencia académica alta, lo que hace que muchos alumnos se quejen terriblemente pero, al final de cuentas, agradecen la formalidad en cuanto a las reglas del juego (P9).

En verdad creo que el alumno, independientemente de la etapa generacional, si le pones las reglas y las respetas, la dinámica de la clase es muy buena y son muy obedientes (P11).

En la parte del rigor académico, yo sí pongo las reglas claras desde el principio, sobre todo en la línea del respeto (P7).

Finalmente, dentro de la metacategoría sobre la figura del profesor, los participantes mencionan dos competencias fundamentales para su labor: el ser practicantes reflexivos y ser generadores de ambientes de aprendizaje. En cuanto a la primera, enfocan la reflexión sobre su práctica docente en diversos aspectos, pero siempre vinculándola a la mejora continua. En lo relacionado a resaltar aspectos personales, hacen referencia a la metamorfosis que van teniendo a lo largo de su trayectoria profesional:

Continuamente estoy pensando en qué clase de persona me estoy convirtiendo en el ejercicio de la docencia (P5).

Pero también a aspectos relacionados con la valoración continua del programa establecido de la asignatura, el cual sirve como fuente para la reflexión:

Tengo mi programa desglosado y rayado con anotaciones, de tal manera que cuando hago el del próximo semestre, lo retomo para mejorarlo, porque obviamente son muchas cosas que se me olvidan. Con esto me doy cuenta de los contenidos que resultan obsoletos y los quito (P4).

Me gusta reflexionar, porque de lo contrario hay una saturación de contenidos y es a veces lo que veo en los alumnos: que les damos gran cantidad de lecturas, artículos y trabajos, pero los dejamos en el hacer y hay una brecha impresionante con el ser (P3).

Además de ser practicantes reflexivos, aluden a la importancia de ser generadores de ambientes que promuevan el aprendizaje, en un clima de cordialidad, respeto y diversión:

Cuando hay un debate en clase, me gusta que tengan una cuestión fundamentada, que no solo participen criticando, sino que nos podamos centrar en un debate, amable, tranquilo y enriquecedor para todos (P10).

Me gusta que la gente que está en el salón de clases, se sienta en un verdadero ambiente de aprendizaje. Me gusta ser un profesor planeado, con estructura, y altamente divertido (P4).

\section{Sobre la práctica docente}

Esta segunda metacategoría se orienta hacia la percepción sobre su actuación profesional, enfocándose a aspectos más técnicos, expresados en seis categorías:

- Planeación.

- Contextualización didáctica.

- Manejo de contenidos.

- Estrategias de enseñanza.

- Variedad de medios didácticos.

- Evaluación. 
En la categoría de planeación, los comentarios pueden agruparse en tres subcategorías: a) la valoración que hacen de esta; b) las orientaciones rectoras, y c) el proceso. En relación con la primera, uno de los participantes expresa la importancia de la planeación como eje articulador:

Desde mis primeros años como profesor, me di cuenta de que sí era importante tener una preparación de clases, porque eso te ordenaba y a los alumnos, también (P6).

Por otro lado, los profesores comparten lo que orienta su planeación, es decir, con base en qué presupuestos la realizan, lo cual va desde la vigencia de los contenidos y la profundidad, pasando por la complejidad y los aspectos formativos, hasta el significado de los mismos:

Empiezo a planear mis clases como cuatro meses antes. Primero lo que es innovador y más sencillo para, después, pasar a lo más complejo y abstracto (P10).

La primera parte del programa es para mí la más importante, porque es la más formativa y la que puede generar un cambio (P5).

Cuando planeo, busco empatar la metodología con los contenidos y pensar las actividades de aprendizaje, de forma que se desarrollen habilidades y siempre busco que todo lo que se esté enseñando tenga un significado práctico (P1).

En cuanto al proceso de planeación, los profesores comparten cómo lo llevan a cabo, siendo reiterativa la idea de centrarse en la organización de los temas y subtemas:

Me gusta partir de un temario ya establecido y complementarlo. Considero, en un primer momento, los tiempos y los temas medulares (P3).

Primero reviso el temario, verifico la secuencia de cada uno de los temas, identifico los que son más profundos que otros -inclusive identifico los temas que son primarios de los secundarios-. Ahí comienza la magia de la planeación (P4).

Conforme al programa de la materia, voy viendo los temas; si es necesario, reacomodo el orden y con base en eso, omito o adiciono. Finalmente, investigo sobre cada uno de los temas (P7).

Sin embargo, otro de los profesores, aunque parte de un temario, considera tanto las estrategias como los recursos que necesita para la impartición de la clase, además de hacer anotaciones a lo largo del semestre. Sobre lo planeado:

Tomo el programa desglosado y empiezo a poner y quitar temas, pero además planteo las estrategias y los recursos que voy a usar, las lecturas que tendrán que realizar los alumnos y trazo, por lo menos cada fin de semana, lo que sí voy a ver en la siguiente (P8).

Una segunda categoría -que los profesores centran en la adaptación de las clases, con base al perfil de los estudiantes- es la contextualización didáctica:

Me considero una persona que me gusta el arte de la enseñanza, es decir, adaptar los conocimientos, la didáctica, los procesos de aprendizaje, al grupo que voy a tener enfrente (P3).

Me paro frente al grupo y comienzo a «escanear[lo]», porque los grupos son totalmente diferentes, cada ser humano es diferente, cada generación es diferente, entonces me lleva una semana o dos adaptar esos contenidos a lo que necesita cada uno de los grupos (P5).

Como doy clases en dos carreras distintas, tengo formas de enseñar diferentes, pues los perfiles de los alumnos así lo demandan, entonces mientras que en Derecho me hablan de «usted», en Comunicación hasta contamos chistes (P6). 
Pasando al manejo de contenidos se encuentran al menos dos criterios de organización: los centrados en contenidos más prácticos que teóricos, y los que buscan hacer asequible el contenido científico:

Intento que no se queden solamente en el concepto, sino que lo «bajemos» a la vida real, donde no se utilizan conceptos, sino que utilizas la parte práctica; entonces intento que, en el transcurso de la clase, por lo menos eso suceda (P10).

En cuanto al manejo de contenidos, busco que sean lo menos teórico posibles. Me interesa mucho más manejar las habilidades; me gusta mucho que sea práctico y compartirles la experiencia que tengo (P1).

Hago un análisis para ver de todo el contenido que hay que dar, lo que a los alumnos les va servir para, entonces, centrarme en buscar las estrategias más adecuadas para pasar del contenido científico al contenido enseñado (P2).

Y sobre las estrategias que emplean durante sus clases, las más recurrentes son: lecturas como fuente de reflexión, intercambio de ideas al interior del grupo y reflexión sobre el aprendizaje. En cuanto a las lecturas, estas sirven de estudio previo:

A veces les pido una lectura previa y luego intento, en cinco o diez minutos en clase, que repasen o que la trabajen con base en los lineamientos que les doy, para sacarle más provecho. Luego ponemos en común las conclusiones (P5).

Siempre les dejo leer -es un recurso que me sirve mucho-, porque cuando llego a clase, los alumnos ya llegan con el antecedente y así el contenido se hace más sencillo (P10).

El intercambio de ideas al interior del grupo surge a partir de estrategias como el manejo de casos, preguntas detonadoras y planteamiento de problemas:

En algunos libros se encuentran casos sencillos con preguntas; eso ayuda a los estudiantes a promover la reflexión de los contenidos (P3).

Soy mucho de construir casos o pequeños problemas, que son párrafos en donde les planteo una situación problemática, de la cual establezco dos o tres preguntas para lograr el objetivo de la clase (P4).

Planteo el tema siempre con preguntas y las llevo al extremo, lo que vuelve bastante divertida la dinámica de la clase (P6).

En cuanto a la reflexión sobre el aprendizaje, los profesores comentan algunas estrategias que van desde el diario hasta el manejo de frases significativas:

Hago con mis alumnos el diario de aprendizaje, donde les pido que identifiquen sus aprendizajes más importantes, las dudas que tienen y algunas sugerencias para que la clase siguiente sea mejor (P1).

Expongo un tema o una inquietud y después les pido que me escriban dos o tres oraciones representativas de lo que más les llamó la atención y que lo comenten entre ellos (P5).

Como otra categoría se encuentra la variedad de medios didácticos que utilizan para atender al principio de novedad en el proceso de enseñanza-aprendizaje $y$, así, promover la participación de los estudiantes:

Me encanta hacer ejercicios, cuestionarios y actividades diversas. Me considero sumamente versátil como profesor. Me gusta variar el estilo (P4). 
Trato de usar variedad de medios didácticos como los casos, el trabajo en equipo y las técnicas grupales (P7).

Por último, dentro de esta metacategoría sobre la práctica docente, se encuentra la categoría de evaluación; los profesores la conciben, por un lado, como retroalimentación, y por el otro, como un espacio de corresponsabilidad entre el estudiante y el profesor. Es decir, la evaluación, entendida como retroalimentación y oportunidad de corrección de trayectoria:

Creo que la evaluación es el elemento más importante, porque es el último momento que tienes para verificar que se está aprendiendo lo que se debe de verificar, y, sobre todo, la evaluación entendida como posibilidad de retroalimentación (P1).

La evaluación para mí no es solamente dar una calificación, porque eso es medición, sino justamente ir trabajando con ellos a lo largo de cada uno de los períodos para que sea una retroalimentación..., para que ellos puedan avanzar (P2).

Cuando dejo tareas, trato de entregarlas a la clase siguiente, porque creo que la retroalimentación es fundamental (P7).

Se distingue la evaluación de la mera medición de conocimientos. Ello involucra tanto al profesor como al estudiante:

Para mí, la evaluación es fundamental en el desarrollo de todo programa académico y de toda persona -que está inmersa en un proceso educativo-, porque mediante ella, tanto el profesor como el alumno, se dan cuenta del proceso, es decir, si cambió, si es diferente, si algo le «dejó»... (P4).

La evaluación es para ambos. El éxito y el fracaso es para ambos: para el alumno y para el profesor; entonces el compromiso que debemos tener los profesores es que siempre sea exitosa (P11).

Una vez llevado a cabo el análisis de la información, a continuación se da paso a la discusión y consideraciones finales de este estudio.

\section{DISCUSIÓN Y CONSIDERACIONES FINALES}

Una vez analizada la información, se infiere que el tipo de cualidades y competencias deseables en un profesor universitario son muy parecidas en las diferentes publicaciones especializadas. Sin embargo, al ser la docencia una profesión compleja y dinámica, el contar con la experiencia -tanto de profesores como de estudiantes- sobre lo que significa ser un buen profesor, resulta sumamente enriquecedora, tanto para el campo de la investigación educativa como para el de la propia docencia.

El tener la posibilidad de acercarse a conocer la práctica de profesores experimentados, como son los participantes en este estudio, ha permitido identificar -desde su experiencia- las características que los hacen ser valorados por sus estudiantes y considerarse ellos mismos como buenos profesores.

Los mismos profesores hacen referencia tanto a aspectos más personales sobre la figura del profesor, como a aspectos más técnicos relacionados con la práctica docente, lo que coincide con el estudio de Alonso (2019), donde señala que un buen profesor universitario cuenta con cualidades personales, profesionales y metodológicas; en donde el dominio de los medios didácticos no juega el papel más importante, sino más bien la preocupación de cuidar 
al otro, de procurarle el mayor bien posible, de cubrir sus necesidades con miras a influir en él y motivarlo hacia la acción educativa (Galbán, 2016; Meza, 2017), mediante la promoción del aprendizaje (Bain, 2007).

Lo anterior lleva a vislumbrar que el que los participantes en esta investigación sean considerados por los alumnos como buenos profesores es, en gran medida, a que colocan en el centro de su actividad profesional a la persona del discente (Meza 2017; Zabalza, 2002), lo cual se manifiesta en la confianza, interés y atención personalizada que les proveen en la tutoría y que se relaciona con la calidad humana, mencionada en el estudio de Alonso (2019).

Además de la tutoría, los profesores enfatizan la importancia que le otorgan al estudio y a la evaluación de los estudiantes, lo que lleva a considerar que estos profesores están alineados con las funciones de la docencia universitaria, mencionadas por Benedito (1992) y Mas (2012).

Siguiendo con la idea de que estos profesores son bien evaluados por los estudiantes, cabe resaltar que una cualidad docente, expresada por Tarabay (2009), es la vocación por la enseñanza, misma que patentizan los participantes en esta investigación, principalmente en su pasión por enseñar, pero también en la confianza y preocupación por los estudiantes, el dominio del contenido, la exigencia académica y el gusto por el estudio.

En relación con las competencias del profesor universitario, mencionadas por Mas (2011) y por Zabalza (2007), ligadas a la planeación, realización y evaluación del proceso didáctico, los participantes hacen referencia a algunas de ellas en la metacategoría sobre la práctica docente, enfatizando lo esencial de la planeación, la contextualización didáctica, el manejo de contenidos, la variedad de medios didácticos y la evaluación. Además de las anteriores, en esta investigación los profesores consideraron como una competencia de su práctica a la reflexión, coincidiendo con lo mencionado por Zabalza (2007), quien habla de la competencia de reflexionar e investigar sobre la enseñanza, y por Mas (2011) que considera el contribuir activamente a la docencia.

Además de ello, se considera un hallazgo interesante el que sea una constante en estos profesores el uso de ciertas estrategias didácticas, orientadas a promover el protagonismo e intercambio de los estudiantes, entre las que se encuentran: manejo de casos, preguntas detonadoras, planteamiento de problemas, así como el uso de diarios y frases significativas, lo cual lleva a intuir que los estudiantes se sienten más identificados y satisfechos con aquellos profesores que promueven ambientes de aprendizaje activo, y tendientes a la vinculación entre la teoría y la práctica.

Es precisamente en este ámbito de las estrategias didácticas activas donde se considera que se encuentran futuras líneas de investigación sobre las cualidades y competencias que abrirán la brecha entre un profesor universitario bien valorado por los estudiantes de otro que no lo es. 


\section{REFERENCIAS}

Alonso, P. (2019). «El perfil del buen docente universitario desde una perspectiva del alumnado». Educação e Pesquisa, 45, 1-22.

Bain, K. (2007). Lo que hacen los mejores profesores de la universidad. Valencia: Publicacions de Univèrsitat de València.

Barrón, M. (2009). «Docencia universitaria y competencias didácticas». Perfiles educativos, 31(125), 76-87.

Benedito, V. (1992). «Formación del profesorado universitario: reflexiones y perspectivas». Revista de Enseñanza Universitaria, 4, 75-100.

Creswell, J. (2002). Educational research. Planning, conducting, and evaluating quantitative and qualitative research. New Jersey: Merrill Prentice Hall.

Durán, A. (2016). «Formación en competencias del docente universitario». Educere, 20(67).

Galbán, S. (2016). Hacia una enseñanza reflexiva. México: Trillas.

Galeano, M. (2004). Diseño de proyectos en la investigación cualitativa. Colombia: Fondo Editorial Universidad EAFIT.

Hernández, R. (2018). «El claustro académico». En F. Llergo y M. Nicolás (direc.), La filosofía educativa de la Universidad Panamericana (pp. 65-74). México: Universidad Panamericana.

Jarauta, B. y Medina, J. (2012). «Saberes docentes y enseñanza universitaria». Estudios sobre educación, 22, 179-198.

Lincoln, Y., Lynham, S. \& Guba, E. (2011). «Paradigmatic controversies, contradictions, and emerging confluences, revisited». In N. Denzin and Y. Lincoln (Eds.), The Sage handbook of qualitative research, 97-128. California: Thousand oaks Sage.

Lincoln, Y. \& Guba, E. (1985). Naturalistic inquiry. California: Thousand oaks Sage.

Mas, O. (2012). «Las competencias del docente universitario: la percepción del alumno, de los expertos y del propio protagonista». Revista de docencia universitaria, 10(2), 299-318.

Mas, O. (2011). «El profesor universitario: sus competencias y formación». Profesorado Revista de currículum y formación del profesorado, 15(3), 195-211.

Merriam, S. \& Tisdell, E. (2016). Qualitative Research: a guide to design and implementation. San Francisco: Jossey-Bass.

Meza, M. (2017). «La dialéctica del amor pedagógico». En R. Casales y N. Blancas (comps.), La esencia del amor, 151-161. México: Tirant Humanidades/UPAEP.

Patton, M. (2015). Qualitative research and evaluation methods. California: Sage.

Pérez, I., Ruiz, C. y Sanz, S. (2014). «El profesor universitario y su función docente». Espacio I+D Innovación más Desarrollo, 3(5), 97-113. doi: 10.31644/ IMASD.5.2014.a05 
Ponz, F. (1996). «Espíritu universitario». En V. García Hoz (comp.), La educación personalizada en la universidad (pp. 81-130). España, Madrid: Rialp.

Robles, B. (2011). «La entrevista en profundidad: una técnica útil dentro del campo antropofísico». Revista Cuicuilco, 18(52), 39-49.

Rodríguez, G., Gil, J. y García, E. (1999). Metodología de la investigación cualitativa. Málaga: Aljibe.

Ruiz, M. y Aguilar, R. (2017). «Competencias del profesor universitario: elaboración y validación de un cuestionario de autoevaluación». Revista Iberoamericana de Educación Superior (RIES), 8(21), 37-65.

Strauss, A. \& Corbin, J. (1998). Basics of qualitative research. Techniques and procedures for developing grounded theory. California: Sage Publications.

Tarabay, F. (2009). «Cualidades docentes universitarias: de la pedagogía a la relación». Revista de Teoría y Didáctica de las Ciencias Sociales, 15, 355-377.

Taylor, S. y Bogdan, R. (1987). Introducción a los métodos cualitativos de investigación. Buenos Aires: Paidós.

Tójar, J. (2006). Investigación cualitativa, comprender y actuar. Madrid: La Muralla.

Zabalza, M. (2002). La enseñanza universitaria. El escenario y sus protagonistas. Madrid: Narcea.

Zabalza, M. (2007). Competencias docentes del profesorado universitario. Calidad y desarrollo profesional. Madrid: Narcea. 